\title{
Una propuesta de localización óptima para un nuevo sitio de disposición final de residuos sólidos no peligrosos para Bogotá, D.C.
}

Proposal for the Optimal Location for a Non Hazardous Waste Site Disposal in Bogotá D.C.

Javier Mauricio González Díaz ${ }^{1}$

Para citar este artículo, utilice el nombre completo así:

González, J. M. (2015). Una propuesta de localización óptima para un nuevo sitio de disposición final de residuos sólidos no peligrosos para Bogotá, D.C. Perspectiva Geográfica, 20(1), 155174.

\section{Resumen}

Para la determinación de la localización óptima de un sitio de disposición final de residuos sólidos, centrada en la teoría de la localización y el análisis multicriterio, se utilizó la Geomática. Se procedió a evaluar la gestión territorial efectuada por el Distrito Capital en la zona actual de operación del Relleno Sanitario Doña Juana, mediante la revisión del expediente 1101-761-1919 Relleno Sanitario Doña Juana. Con el fin de examinar los factores que condicionan la localización óptima de un nuevo sitio de disposición final, se definieron los criterios técnicos, ambientales y sociales que delimitan las zonas aptas para la localización de este tipo de instalaciones, con base en lo establecido en el Decreto 838 de 2005. Finalmente, se generan diferentes escenarios $\mathrm{y}$, por medio de la evaluación multicriterio, se propone una

1 Ingeniero ambiental y sanitario. Especialista en Evaluación del impacto ambiental de proyectos. Magíster en Geografía, convenio UPTC-IGAC. Docente Universidad de La Salle. javigonzalez@unisalle.edu.co zicogonzalez@gmail.com 
localización óptima de un nuevo sitio para la disposición final de residuos sólidos para Bogotá.

Palabras clave: gestión territorial, geomática, localización, multicriterio, residuos sólidos.

\begin{abstract}
To determine the optimal location for a non-hazardous waste site with focus on the theory of localization and multi-criteria analysis, Geomatics was used. We proceeded to assess the territorial management by the Capital District in the current area of operation of "Doña Juana" Landfill by reviewing the record 1101-761-1919 "Doña Juana" Landfill. In order to examine the factors that determine the optimal location of a new disposal site, technical, environmental and social criteria defining areas suitable for the location of such facilities were delineated, based on the provisions of the Decree 838 of the year 2005. Lastly, different situations were generated and through multicriteria evaluation an optimal location for the disposal of solid waste in Bogotá was proposed.
\end{abstract}

Keywords: territorial management, geomatics, location, multicriteria, solid waste. 


\section{Introducción}

Con la promulgación de la Constitución Política de Colombia, el país dio pasos fundamentales en la protección de los recursos naturales; se establecieron principios para el cuidado y aprovechamiento del medio ambiente, parte de ellos desarrollados en función del ordenamiento territorial. Por tanto, se hizo necesario la construcción de nuevas normas, tales como la Ley 99 de 1993, sobre el Sistema Nacional Ambiental, SINA, la Ley 142 de 1994, de Servicios Públicos, la Ley 388 de 1997, sobre ordenamiento territorial, el Decreto 1713 de 2002 (hoy derogado por el Decreto 2981 de 2013) que reglamentó la prestación del servicio público de aseo; el Decreto 838 de 2005 , sobre disposición final de residuos sólidos, el Decreto 4741 de 2005 , referente a la gestión integral de residuos peligrosos, y el Decreto 2041 de 2014, que establece el licenciamiento ambiental para proyectos, obras o actividades que modifiquen el entorno natural, entre otras, que permitieron organizar la gestión territorial y ambiental para el manejo de los residuos sólidos.

No obstante, cada día se insiste, con mayor frecuencia, en el apro- vechamiento de los residuos, y la tendencia actual es la disminución de la fracción de aquellos destinados a rellenos sanitarios, con lo cual se aumenta el incremento en las cifras correspondientes al reciclaje y el compost. La meta de muchos países, en particular los europeos, es, en un futuro, no utilizar rellenos sanitarios (Noguera \& Olivero, 2010).

En Colombia, el aprovechamiento de los residuos no ha sido significativo, por lo cual el Estado, en su gestión territorial, privilegia la disposición final de los residuos en rellenos sanitarios. El Relleno Sanitario Doña Juana presenta problemas diversos, tales como la proliferación de olores ofensivos, vectores como moscas y roedores, debido a la exposición de basuras que no son cubiertas a tiempo, y volúmenes de lixiviados superiores a la capacidad hidráulica de la planta de tratamiento de los mismos. Por causa de estos problemas, las comunidades aledañas se han visto afectadas; se reporta, además, tierras menos fértiles e infección del ganado (Noguera \& Olivero, 2010).

Es importante destacar cómo el contexto social, que envuelve 
la localización de rellenos sanitarios, hace difícil que los mecanismos de compensación puedan efectivamente disminuir la oposición de las comunidades potencialmente afectadas (Claro, 2001). Como consecuencia de lo anterior, la localización de nuevas instalaciones de disposición de residuos sólidos domiciliarios es un esfuerzo complejo y difícil, en el que deben coordinarse factores técnicos, ambientales, económicos, sociales y políticos (Claro, 2001), en donde resulta clave el papel de los fundamentos teórico-metodológicos que aporta la geografía.

Como hipótesis del problema se plantea que el uso de la Geomática y la gestión territorial, apoyados en los criterios técnicos, ambientales, sociales y geográficos que establece el Decreto 838 de 2005, permitirán proponer una localización óptima para la disposición final de los residuos sólidos no peligrosos en Bogotá, como consecuencia del agotamiento de la vida útil del Relleno Sanitario Doña Juana. Con base en lo anterior, la presente investigación, bajo los lineamientos de la planeación, ordenación y gestión territorial, se trazó como propósito central

proponer una localización óptima de un nuevo sitio de disposición final de residuos sólidos mediante la interpretación y valoración de los efectos socio-espaciales y territoriales para la localización de infraestructura de servicios y equipamientos públicos.

\section{Planteamiento del problema}

El territorio y la región son expresiones de la espacialización del poder y de las relaciones de cooperación o de conflicto que de ella se derivan (Gómez \& Delgado Mahecha, 1998). Para hablar de espacio geográfico es preciso centrarse en lo que define Milton Santos (1997) citado por (Gómez \& Delgado Mahecha, 1998), en donde establece que es un conjunto indisociable de objetos y de sistemas de acciones. Los sistemas de objetos no ocurren sin los sistemas de acciones y estos últimos no suceden sin los primeros. El espacio es construido históricamente; agrega Santos (1997) que el espacio geográfico, hoy, es un sistema de objetos cada vez más artificiales, provocados por sistemas de acciones igualmente imbuidas de artificialidad, y cada vez más tendientes a fines extraños al lugar y a sus habitantes. Continúa 
Santos afirmando que, actualmente, se vive en una época en que el número de objetos del espacio geográfico se ha multiplicado exponencialmente: "en los últimos cuarenta años se vieron nacer sobre la faz de la tierra más objetos que en los anteriores cuarenta mil años" (p. 122). Localizar y operar un relleno sanitario en cualquier espacio geográfico hace parte de lo que afirma Santos (1997), porque este es un sistema artificial que modifica tanto la topografía como el entorno natural y se convierte en un objeto extraño al lugar y a sus habitantes.

Estos conflictos de territorio, que van desde lo ambiental hasta lo social, son generados por actividades no deseables y que se localizan en territorios que los repelen. (Gómez \& Delgado Mahecha, 1998). La capacidad y alcance de la actividad espacial es desigual y convergente en los lugares en donde se genera una geografía del poder caracterizada por la desigualdad, la fragmentación, la tensión y el conflicto. Entonces, el territorio se construye a partir de la actividad espacial de agentes que operan en diversas escalas (Massey, 1995, citado por Gómez \& Delgado 1998); la actividad espacial se refiere a la red espacial de relaciones y actividades, de conexiones espaciales y de localizaciones con las que opera un agente determinado, lo que contribuye a reducir sustancialmente la calidad de vida de los habitantes que se ubican cerca de un relleno sanitario.

Una de estas demandas ambientales (por usos del territorio para localizar instalaciones no deseables con las consecuentes afectaciones a los recursos naturales) está asociada a la generación creciente de los residuos sólidos, ya que la preocupación por los residuos generados en los centros urbanos parte de las consideraciones de tipo higiénico, sanitario y geográfico. El crecimiento poblacional sin control alguno, las migraciones internas producto de los desplazamientos forzados o no forzados y el atractivo de las capitales como centro de oportunidades laborales y de desarrollo de "una mejor calidad de vida" ha ocasionado que, desde los años 70, ciudades como Bogotá hayan tenido un crecimiento poblacional significativo, lo cual incluye el aumento y proliferación de actividades terciarias especializadas; como consecuencia de esto, se presenta el incremento en el consumo de productos, que ha traí- 
do como consecuencia un aumento significativo en la generación de residuos, lo que conlleva tomar decisiones drásticas para acceder a nuevos sitios de disposición final, con los impactos ambientales, sanitarios, sociales y, por supuesto, geográficos que esto produce.

En este contexto, las decisiones en políticas sobre la implantación de usos del suelo no deseables (plantas químicas, centrales nucleares o rellenos sanitarios, entre otros) se encuentran frecuentemente ante una reacción pública negativa, muchas veces violenta, debido a que generan en la población, potencialmente afectada, un sentimiento de injusticia al pensar que cae sobre ellos toda la carga negativa de una actividad planeada para beneficio del resto de la sociedad. La búsqueda de localizaciones de servicios comunitarios estratégicos para la ciudad, tales como: incineradoras, vertederos o depósitos de seguridad de residuos tóxicos y peligrosos ha generado, hasta ahora, un fuerte rechazo popular. Por tanto, el problema tiene un importante componente espacial. (Sendra, Muñoz, Durán, \& García, 2000).

Para dar respuesta al sinnúmero de problemas derivados de la práctica mencionada y a muchos otros relacionados con la ubicación de instalaciones de servicios comunitarios estratégicos, la gestión del territorio en Colombia se ha tratado de llevar a cabo a partir de un ordenamiento territorial (Ley 388 de 1997), en donde todos los municipios deben ordenar y planear el territorio bajo su jurisdicción. Esta Ley establece el conjunto de principios, instrumentos y disposiciones sobre planeación y gestión territorial, con el fin de lograr un ordenamiento físico que promueva el desarrollo socioeconómico en armonía con la naturaleza; igualmente, incorpora de manera definitiva tanto la dimensión ambiental como la territorial en la planeación del país (Hernández-Gómez, Rojas-Robles, \& Sánchez-Calderón, 2013).

Como parte de la solución de los problemas planteados en la Política de residuos Sólidos, el Consejo Nacional de Política Económica y Social, emitió un documento, en 2008, que se denominó CONPES 3530 (2008), en donde se establecieron los lineamientos y estrategias para fortalecer el servicio público de aseo en el marco de la gestión integral de residuos sólidos. Allí, se diagnosticó la insuficiencia de sitios técnicamente ade- 
cuados para la disposición final de residuos sólidos, pues afirma que, en Colombia, en una muestra de 1.088 municipios, se genera, aproximadamente, 25.079 toneladas/ día de residuos; de los cuales, el 31\% (388 municipios) disponen sus residuos de forma inadecuada: 284 botaderos, 19 enterramientos, 7 quemas y 8 en cuerpos de agua (Conoes 3530, 2008).

A pesar de que el comportamiento, en los últimos años, ha sido positivo en materia de sitios de disposición final, aún existen municipios con capacidad técnica y financiera que no los han implementado o zonas donde es técnicamente viable la implementación de esquemas regionales para la disposición final, y aún persisten objeciones por parte de las autoridades municipales para la localización del sitio. Un ejemplo de ello es el Relleno Sanitario Doña Juana que cuenta, actualmente, con una extensión de 594 ha. de terreno, de las cuales solamente el $22 \%$ se destina para la disposición final. Las demás hectáreas son utilizadas como zonas de amortiguamiento y protección ambiental (Contraloría de Bogotá, D.C., 2012). Los residuos afectan, en general, todas las actividades, personas y espacios, convirtién- dose en un problema no solo por lo que representan en términos de recursos abandonados (porque no se aprovechan o no se reciclan, y por supuesto no se reincorporan al ciclo productivo), sino por la creciente incapacidad para encontrar lugares que permitan su acomodo correcto desde el punto de vista ambiental (Molina, 2008).

\section{Metodología}

Para el cumplimiento de los objetivos planteados, se diseñó una metodología documental, descriptiva y correlacional; se utilizó el método general de análisis multicriterio. La metodología documental consiste en la recopilación y selección de información por medio de consulta, la cual es necesaria para la investigación planteada; la metodología descriptiva consiste en la evaluación y recolección de datos sobre diversos conceptos, aspectos, dimensiones o componentes del objeto de investigación. Por su parte, el método general de análisis multicriterio aporta la diversidad de factores que se logran integrar en el proceso de evaluación; por tanto, según lo afirmado por Contreras \& Pacheco (2007, citado por Grajales-Quintero, Serrano-Moya, Hahn Von-H, 2013), esta per- 
mite transformar las mediciones y percepciones en una escala única para comparar los elementos y establecer órdenes de prioridad que permitan agregar los efectos de un proyecto en una métrica común.

El método de análisis general multicriterio permite la decisión más flexible sobre una localización óptima para la disposición de los residuos sólidos, porque facilita descomponer un problema complejo en partes más simples; genera un modelo jerárquico que, básicamente, contiene tres niveles: objetivos, criterios y alternativas, jugando un papel vital como herramienta de planeación territorial (Nijkamp et al., 1990 citados por Grajales-Quintero, Serrano-Moya, Hahn Von-H, 2013). Los reportes de datos geográficos son obtenidos de diversas fuentes; en la Tabla 1 se especifica la descripción del análisis de información necesaria para poder aplicar los criterios establecidos en el Decreto 838 de 2005.

Tabla 1. Captura de información

\begin{tabular}{|c|c|c|c|}
\hline INFORMACIÓN & FUENTE & ESCALA & USO - CRITERIO \\
\hline $\begin{array}{c}\text { Shapefile Clase } \\
\text { de suelo (rural, } \\
\text { expansión urbana, } \\
\text { urbano) }\end{array}$ & $\begin{array}{c}\text { PLANEACIÓN } \\
\text { DISTRITAL }\end{array}$ & $1: 10.000$ & $\begin{array}{c}\text { Criterio ocupación actual del } \\
\text { área, criterio distancia entre el } \\
\text { perímetro urbano respecto del } \\
\text { área para la disposición final } \\
\text { y criterio distancia al suelo } \\
\text { urbano. }\end{array}$ \\
\hline $\begin{array}{c}\text { Shapefile áreas de } \\
\text { manejo especial }\end{array}$ & IGAC & $1: 100.000$ & $\begin{array}{c}\text { Criterio ocupación actual del } \\
\text { área y Áreas pertenecientes al } \\
\text { sistema de parques nacionales. }\end{array}$ \\
\hline $\begin{array}{c}\text { Shapefile parque } \\
\text { ecológico distrital } \\
\text { de montaña }\end{array}$ & IGAC & $1: 100.000$ & $\begin{array}{c}\text { Criterio ocupación actual del } \\
\text { área y Áreas pertenecientes al } \\
\text { sistema de parques nacionales. }\end{array}$ \\
\hline $\begin{array}{c}\text { Shapefile parque } \\
\text { nacional natural }\end{array}$ & IGAC & $1: 100.000$ & $\begin{array}{c}\text { Criterio ocupación actual del } \\
\text { área y Áreas pertenecientes al } \\
\text { sistema de parques nacionales. }\end{array}$ \\
\hline $\begin{array}{c}\text { Shapefile reserva } \\
\text { forestal distrital }\end{array}$ & IGAC & $1: 100.000$ & $\begin{array}{c}\text { Criterio ocupación actual del } \\
\text { área y Áreas pertenecientes al } \\
\text { sistema de parques nacionales. }\end{array}$ \\
\hline
\end{tabular}




\begin{tabular}{|c|c|c|c|}
\hline INFORMACIÓN & FUENTE & ESCALA & USO - CRITERIO \\
\hline $\begin{array}{c}\text { Shapefile } \\
\text { santuario distrital } \\
\text { de fauna y flora }\end{array}$ & IGAC & $1: 100.000$ & $\begin{array}{l}\text { Criterio ocupación actual del } \\
\text { área y Áreas pertenecientes al } \\
\text { sistema de parques nacionales. }\end{array}$ \\
\hline Shapefile vías & IGAC & 1:100.000 & $\begin{array}{l}\text { Criterio Accesibilidad vial } \\
\text { y criterio disponibilidad de } \\
\text { material de cobertura. }\end{array}$ \\
\hline $\begin{array}{l}\text { Shapefile Curvas } \\
\text { de nivel cada } 100 \\
\text { mts. }\end{array}$ & $\begin{array}{l}\text { IGAC - PHP } \\
2014\end{array}$ & $1: 100.000$ & $\begin{array}{l}\text { Mapa de Pendientes del } \\
\text { terreno para el criterio de } \\
\text { condiciones de topografía. }\end{array}$ \\
\hline $\begin{array}{l}\text { Shapefile } \\
\text { Densidad } \\
\text { poblacional }\end{array}$ & DANE & $\mathrm{Hab} / \mathrm{km} 2$ & $\begin{array}{l}\text { Criterio densidad poblacional } \\
\text { en el área. }\end{array}$ \\
\hline $\begin{array}{c}\text { Shapefile } \\
\text { hidrología urbana }\end{array}$ & IDECA & $1: 10.000$ & $\begin{array}{c}\text { Criterio distancia a cuerpos } \\
\text { hídricos. }\end{array}$ \\
\hline $\begin{array}{l}\text { Shapefile Drenajes } \\
\text { rural }\end{array}$ & $\begin{array}{l}\text { IGAC - PHP } \\
2014\end{array}$ & 1:100.000 & $\begin{array}{c}\text { Criterio distancia a cuerpos } \\
\text { hídricos. }\end{array}$ \\
\hline Mapa de vientos & $\begin{array}{l}\text { IDEAM y } \\
\text { FOPAE }\end{array}$ & $\begin{array}{c}\text { Sin } \\
\text { información }\end{array}$ & $\begin{array}{c}\text { Criterio dirección de los } \\
\text { vientos. }\end{array}$ \\
\hline $\begin{array}{l}\text { Shapefile de } \\
\text { suelos }\end{array}$ & IGAC & $1: 100.000$ & $\begin{array}{l}\text { Criterio calidad del material de } \\
\text { cobertura. }\end{array}$ \\
\hline $\begin{array}{l}\text { WMS mosaico de } \\
\text { catastro Bogotá } \\
2009\end{array}$ & $\begin{array}{l}\text { http://imagenes. } \\
\text { catastrobogota. } \\
\text { gov.cO }\end{array}$ & $\begin{array}{l}\text { Excelente } \\
\text { nivel de } \\
\text { detalle. Sin } \\
\text { información } \\
\text { de escala } \\
\end{array}$ & $\begin{array}{c}\text { Criterio disponibilidad de } \\
\text { material de cobertura, criterio } \\
\text { geoformas del área respecto al } \\
\text { entorno y reconocimiento de } \\
\text { la zona. }\end{array}$ \\
\hline $\begin{array}{c}\text { Shapefile } \\
\text { pista aterrizaje } \\
\text { aeropuertos }\end{array}$ & IGAC & 1:100.000 & $\begin{array}{l}\text { Restricción proximidad a } \\
\text { aeropuertos. }\end{array}$ \\
\hline $\begin{array}{l}\text { Remoción en } \\
\text { masa }\end{array}$ & $\begin{array}{l}\text { PLANEACIÓN } \\
\text { DISTRITAL }\end{array}$ & $1: 100.000$ & Restricción Áreas instables. \\
\hline $\begin{array}{l}\text { Shapefile } \\
\text { zonificación } \\
\text { sísmica }\end{array}$ & SIGOT & $\begin{array}{l}\text { Sin } \\
\text { información }\end{array}$ & $\begin{array}{l}\text { Restricción zonas de riesgo } \\
\text { sísmico alto. }\end{array}$ \\
\hline Shapefile de aves & $\begin{array}{l}\text { PLANEACIÓN } \\
\text { DISTRITAL }\end{array}$ & $\begin{array}{c}\text { Sin } \\
\text { información }\end{array}$ & $\begin{array}{l}\text { Prohibición de hábitat } \\
\text { naturales críticos. }\end{array}$ \\
\hline $\begin{array}{l}\text { Shapefile de } \\
\text { Mamíferos }\end{array}$ & $\begin{array}{l}\text { PLANEACIÓN } \\
\text { DISTRITAL }\end{array}$ & $\begin{array}{c}\text { Sin } \\
\text { información }\end{array}$ & $\begin{array}{l}\text { Prohibición de Hábitat } \\
\text { naturales críticos. }\end{array}$ \\
\hline
\end{tabular}




\begin{tabular}{|c|c|c|c|}
\hline INFORMACIÓN & FUENTE & ESCALA & USO - CRITERIO \\
\hline $\begin{array}{c}\text { Shapefile Fallas } \\
\text { Geológicas }\end{array}$ & SIGOT & $1: 200.000$ & $\begin{array}{c}\text { Prohibición áreas con fallas } \\
\text { geológicas. }\end{array}$ \\
\hline $\begin{array}{c}\text { Shapefile Fuente } \\
\text { de captación de } \\
\text { consumo }\end{array}$ & IGAC & $1: 100.000$ & $\begin{array}{c}\text { Prohibición fuentes } \\
\text { superficiales. }\end{array}$ \\
\hline $\begin{array}{c}\text { Shapefile } \\
\text { zonificación } \\
\text { climática }\end{array}$ & SIGOT & Sin & información \\
\hline $\begin{array}{c}\text { Centros poblados } \\
\text { Shapefile } \\
\text { Geología }\end{array}$ & INGEA & $1: 20.000$ & Componente social. \\
\hline $\begin{array}{c}\text { Shapefile Zonas } \\
\text { hidrogeológicas }\end{array}$ & SIGOT & $\begin{array}{c}\text { Sin } \\
\text { información }\end{array}$ & Componente hídrico. \\
\hline
\end{tabular}

Fuente: La información de la cartografía base se tomó de la Secretaría Distrital de Planeación de Bogotá, de acuerdo con lo aprobado en el POT en escala 1:100.000.

\section{Resultados}

Con base en ese análisis de gestión territorial realizado al área actual de disposición final de residuos sólidos en Doña Juana se pudo establecer que:

La afectación sobre los cuerpos de agua superficial, ubicados en el área de influencia del Relleno Sanitario Doña Juana, es alta debido, principalmente, a los vertimientos de lixiviados descargados a las quebradas Yerbabuena y el Botello, y al propio río Tunjuelo, pues los parámetros de calidad establecidos por el Decreto 1594 de 1984 y el Acuerdo 43 de 2006 son superados en estos cuerpos de agua en un
$86 \%$ en cuanto al parámetro de DBO, y $100 \%$ en cuanto al parámetro de $\mathrm{SST}^{2}$ Con respecto al componente atmosférico, la gestión territorial no ha sido la suficiente. Las concentraciones y el comportamiento diario de PST (Partículas Suspendidas Totales) y PM10 (Material Particulado menor a 10 micras), no se ha cumplido en su totalidad, pues las acciones realizadas por el operador, como riego de agua en las vías destapadas en el interior del proyecto, el control técnico mecánico de los camiones recolectores y la utilización de cobertura intermedia en el frente de trabajo, no han sido lo

2 Expediente 1101-761-1919 Relleno Sanitario Doña Juana. 
suficientemente correctivas, ya que las estaciones ubicadas dentro del relleno sanitario (Zona VII y Zona VIII) reportan concentraciones que superan los máximos diarios permisibles en un $11,5 \%$ para PST y en un $25 \%$ para PM10 $0^{3}$. Igualmente, otro de los problemas de gestión territorial y ambiental en el tema de calidad de aire que no ha sido posible mitigar corresponde a la generación de olores ofensivos tanto en los frentes de trabajo como en el área de influencia directa (barrios Mochuelo Bajo y Alto, Tenerife, Aurora 1 y 2, Marichuela, Barranquitos, Quintas, Plan Social, entre otros); se ha generado inconformidades y quejas que, en la fecha, no se han resuelto efectivamente.

Con respecto al área de influencia de la operación del sitio de disposición final actual se puede establecer que los impactos generados por la operación del relleno sanitario no solo afectan el área de disposición de residuos, sino que se extienden a más de 7 $\mathrm{km}$. para los componentes sociales y atmosféricos, y una extensión igual a la cuenca del Río Tunjuelo para el componente hídrico; es

3 Expediente 1101-761-1919 Relleno Sanitario Doña Juana. CAR de Cundinamarca. significativa la afectación sobre el territorio. Dentro de los hallazgos encontrados en la evaluación del expediente, se pudo determinar que existe un incumplimiento reiterado en el Plan de Manejo Ambiental y el plan de seguimiento y control, aprobados mediante la Resolución 2791 del 2008, por medio de la cual se otorgó la Licencia Ambiental, puesto que no se ha efectuado los monitoreos respectivos a los contaminantes $\mathrm{NO}_{x}, \mathrm{SO}_{x}, \mathrm{CO} y$ $\mathrm{O}_{3}$, tanto en el interior como en el exterior del sitio de disposición final, y no se realizaron los respectivos monitoreos de vectores con la frecuencia establecida, por lo que no es posible establecer, claramente, cuál es el estado del territorio y de la calidad ambiental para el recurso aire. En la cultura capitalista, el desarrollo lleva implícita la noción de crecimiento y acumulación, enmarcada en la búsqueda de acceso inescrupuloso a mercados cautivos, materia prima abundante, mano de obra barata, mentes dóciles y cuerpos indisciplinados (De Souza, 2010, citado por Massiris A., 2012).

Para la localización de nuevas áreas para el enterramiento de residuos se establece como criterios, los definidos en el Decreto 838 de 
2005, los cuales corresponden a: ocupación actual del área, condiciones de topografía, distancia entre el perímetro urbano, densidad poblacional en el área, distancias a cuerpos hídricos, prohibición de fuentes superficiales, prohibición de áreas con fallas geológicas, prohibición de áreas pertenecientes al sistema de parques nacionales naturales, restricción de distancia al suelo urbano, restricción de proximidad a aeropuertos, restricción de áreas inestables, restricción de zonas de riesgo sísmico alto, capacidad, accesibilidad vial, condiciones del suelo y topografía, disponibilidad de material de cobertura, incidencia en la congestión de tráfico en la vía principal, dirección de los vientos, geoformas del área respecto del entorno, prohibición fuentes subterráneas y prohibición de localización de hábitat naturales críticos. Con el fin de establecer las áreas potenciales para localizar un nuevo sitio de disposición final de residuos sólidos, se hace necesario estimar tanto las proyecciones de población para la ciudad como la proyección de residuos sólidos para los próximos 30 años. Lo anterior, dados los métodos establecidos en el RAS Título B (Reglamento Técnico del Sector Agua Potable y Saneamiento Básico).
Ahora bien, teniendo en cuenta el consumo creciente de productos y su consecuente generación progresiva de residuos sólidos se establece una producción percápita para el año 2015 de 0,894 Kg/ Hab-Día, con un crecimiento sostenido de $0,7 \%$ de aumento de consumo anual. De acuerdo con los diseños del relleno sanitario tipo terraza, se tendrá que para todo el horizonte del proyecto (30 años, de acuerdo con lo establecido en el Decreto 838 de 2005), se generarán 229.884.997,74 toneladas de residuos, con volumen esperado de 255.427.775,26 $\mathrm{m}^{3}$ en 30 años de vida útil del proyecto, con una densidad promedio esperada $900 \mathrm{Kg} /$ $\mathrm{m}^{3}$. De acuerdo con los diseños, se estima una altura desde la base natural del suelo de 45 metros, reportando un área mínima necesaria de enterramiento de 638, 57 ha. Sin embargo, el área deberá ser igual o mayor a 1.5 veces la producción de residuos sólidos producidos en treinta años (MAVDT, 2005); entonces, como resultado de esta multiplicación, se necesita un área mínima necesaria de 851,43 ha. Es de señalar que las áreas óptimas que se establecen en la presente investigación (851,43 ha.) corresponden a áreas donde las actividades de aprovechamiento de los residuos 
orgánicos y reciclables, mediante la incorporación de residuos sólidos al ciclo productivo, no se realizarán, y que como consecuencia se hace necesario enterrar todos los residuos que se producen, con lo que se fija el peor de los escenarios de gestión de residuos sólidos tal como sucede en la actualidad. Una vez efectuada la respectiva evaluación, se obtuvo como resultado el mapa de la sumatoria de puntajes de los criterios analizados a partir de la aplicación de los sistemas de información geográfica y del análisis espacial; el resultado: cinco áreas que se presentan en la Tabla 2 para, finalmente, escoger a Nazareth por ser el área que menos impacto recibirá por la instalación de este tipo de actividades no deseables (ver Figura 1.).

Tabla 2. Áreas seleccionadas

\begin{tabular}{|l|r|c|}
\hline \multicolumn{1}{|c|}{ Área seleccionada } & Área (ha.) & Puntaje \\
\hline 1. Guaymaral & $1.442,39$ & 207 \\
\hline 2. Suba & $1.068,60$ & 205 \\
\hline 3. Chisacá & 693,96 & 262 \\
\hline 4. Nazareth & $1.215,860$ & 210 \\
\hline 5. Regadera & 663,43 & 305 \\
\hline
\end{tabular}

Fuente: Elaboración propia. 


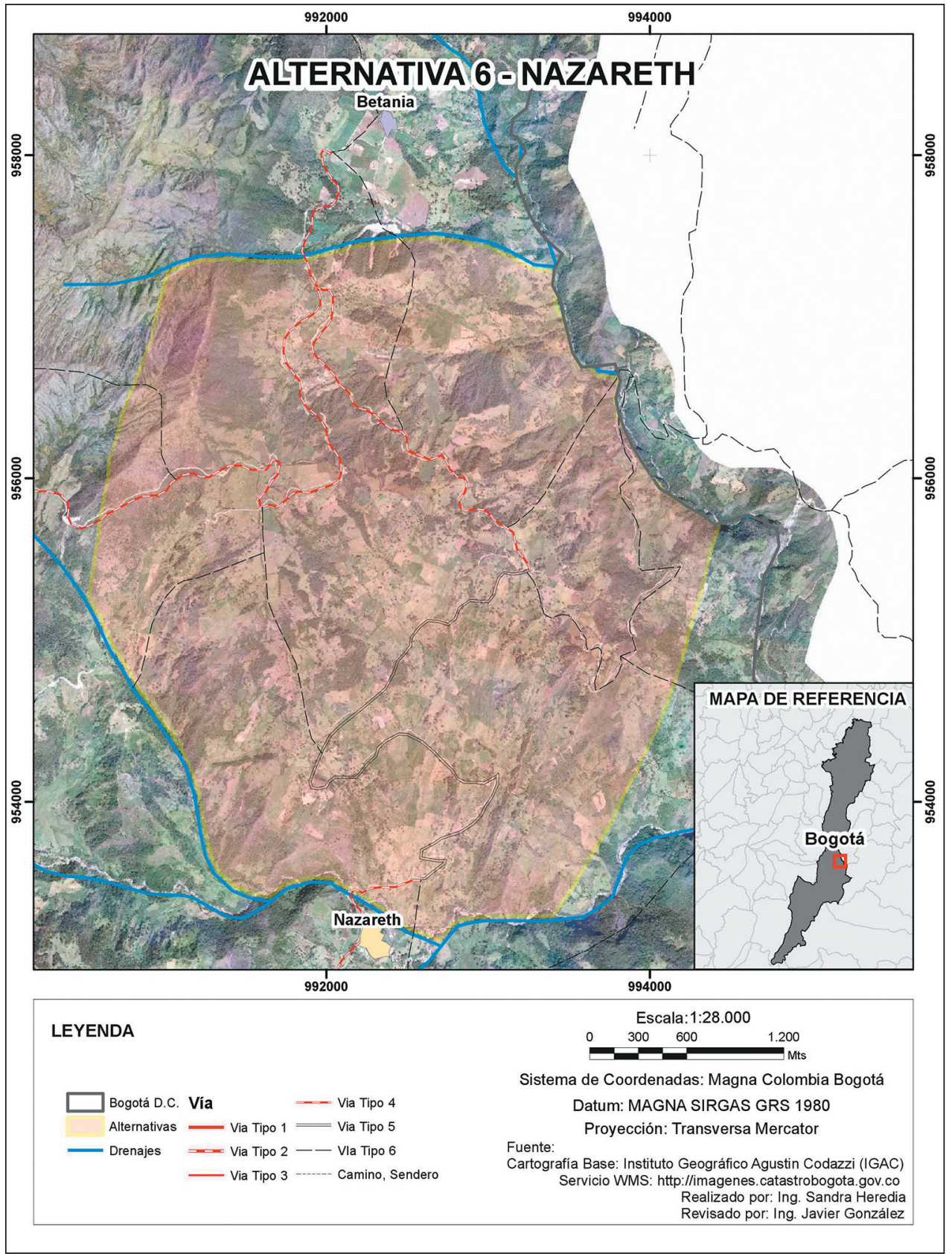

Figura 1. Propuesta de óptimo locacional.

Fuente: Elaboración propia. 


\section{Conclusiones}

A partir de la evaluación efectuada sobre los diferentes territorios, mediante la aplicación de los criterios definidos en el Decreto 838 de 2005, como herramienta fundamental para la toma de decisiones en Colombia para la selección de territorios susceptibles de ser utilizados como sitios para la disposición final de residuos sólidos, y con ayuda de la geomática para la captura, el análisis e interpretación de información geográfica del territorio seleccionado para la toma de decisiones, se puede establecer que el área de evaluación corresponde a un $44 \%$, utilizando información geográfica como base para realizar la representación del $48 \%$ de las variables. Lo anterior, permite producir cartografía exclusiva, de acuerdo con los lineamientos establecidos en el Decreto, con el fin de efectuar la superposición de cartografías y, con base en los múltiples criterios, tomar la mejor decisión (Ambiental, Sanitaria, Social y de localización).

Con base en lo anterior, a partir del análisis espacial y la evaluación multicriterio, se definieron cinco posibles áreas dentro de la jurisdicción del Distrito Capital, que arro- jaron como mínimo los 600 puntos exigidos en el Decreto 838 de 2005, como áreas para ser evaluadas en un posterior estudio de impacto ambiental, como herramienta para la toma de decisiones para el otorgamiento de la Licencia Ambienta $1^{4}$. Es importante aclarar que no solo se trata, en esta investigación, de determinar unas posibles áreas para localizar un nuevo sitio de disposición final de residuos sólidos no peligrosos para Bogotá, pues cada una de esas posibles áreas traen consecuencias ambientales, sanitarias, sociales y geográficas por ser una instalación no deseable. Es por esto que la alternativa uno, denominada Guaymaral, y la dos, Suba, son descartadas porque en la evaluación preliminar el componente social es muy alto en las comunidades colindantes con el proyecto, y por estar incluida dentro del área correspondiente a la zona de manejo y preservación del Río Bogotá.

La alternativa correspondiente a Chisacá, en su área de influencia directa, afecta a cuatro veredas, un

4 Ley 99 de 1993. Por la cual se crea el Ministerio del Medio Ambiente, se reordena el Sector Público encargado de la gestión y conservación del medio ambiente y los recursos naturales renovables, se organiza el Sistema Nacional Ambiental, SINA, y se dictan otras disposiciones. 
centro poblado y la vía principal que comunica la zona urbana con el Páramo de Sumapaz. La alternativa correspondiente a la zona denominada Regadera, por su importancia hídrica, presiona directamente el nacimiento de, por lo menos, 6 cuerpos de agua y toda la zona de recargas de acuíferos; resulta una prohibición de localización, según el Decreto 838 del 2005. Es evidente que cualquier área seleccionada causa impactos ambientales para este tipo de proyectos; sin embargo, la zona donde fue posible establecer una localización para construir y operar este tipo de proyectos con la magnitud e importancia necesaria, como parte final en la prestación del servicio público de aseo, se denomina $\mathrm{Na}-$ zareth.

Esta área se localiza en el Centro poblado de Nazareth; a pesar de que es un área con influencia de especies en peligro de extinción, ofrece criterios con mayores posibilidades para emplazar un relleno sanitario para los próximos 30 años, teniendo en cuenta que en un período no menor a 15 años, los comportamientos y actitudes de los ciudadanos de hoy y del Estado para reducir la generación creciente de residuos, no se dará. Actual- mente, las campañas de educación ambiental, de cambios comportamentales y de actitud, sumado a que el programa de Basura Cero impulsado por la actual administración distrital no ha arrancado con los programas propuestos, $\mathrm{y}$ en la fecha, por solo nombrar alguno de ellos, el programa de ruta de recolección selectiva en toda la ciudad ni siquiera se ha lanzado, mucho menos se espera que en tan corto tiempo se implemente, lo que conllevará necesitar un área para disponer todos los residuos que se generarán en los próximos 30 años.

El área propuesta (Nazareth) está establecida pensando en que la ciudad no hará esfuerzos ingentes por iniciar procesos de separación en la fuente en los sectores residencial, comercial, institucional e industrial, implementación futura de rutas selectivas con cobertura del $100 \%$ de la ciudad, sistemas de aprovechamiento de los residuos orgánicos con técnicas que ya están $100 \%$ probadas en otras latitudes, no solo de América, sino en el mundo, y por supuesto la reincorporación de los materiales reciclables al ciclo productivo. Es decir, que el escenario propuesto en esta alternativa está determinado para enterrar todos los residuos 
que se produzcan sin pensar que habrá modificaciones de actitudes, comportamientos y de políticas por parte del Estado, favorables para la gestión y aprovechamiento de los residuos a corto plazo en la ciudad.

Si lo anterior se diera, se podría muy rápidamente, maximizar la cantidad de residuos que realmente pueden ser aprovechados y reciclados, y solo una mínima parte, la que realmente es basura, podrá ser enterrada o, por qué no, utilizar otras alternativas de destrucción y eliminación como la incineración, pues en países europeos, como en Suecia, la política medioambiental fomenta el reciclaje y la incineración como opciones primarias en la gestión de residuos; como última opción, recurren al relleno sanitario. Es de exaltar la gestión territorial solidarizada que realiza el Estado sueco y sus ciudadanos, solo basta con conocer que la cantidad de residuos que van a los rellenos ha decrecido de una manera sorprendente: de 6,1 millones de toneladas en 1994 a 1,9 millones de toneladas en el 2005, una caída de $68 \%$, en donde solo el $4,8 \%$ eran residuos sólidos domésticos (Calderón Pradilla, 2010).
Massiris, soportado en De Souza (2010), plantea que el desarrollo territorial está mal concebido, si se mira desde una perspectiva holística: el consumo sin control, el uso indiscriminado de recursos naturales para satisfacer necesidades banales cada vez mayores hace que la sociedad se convierta en mentes dóciles (compran y consumen todo lo que ven sin evaluar su responsabilidad, como ciudadanos, en la generación de residuos sólidos) y, por supuesto, totalmente indisciplinadas, pues no existe el autocontrol en lo que se adquiere, en lo que se consume y en lo que se produce.

Massiris (2012) precisa que, en algunas comunidades indígenas suramericanas, no existe la noción del desarrollo como expresión de un estado anterior y posterior; es decir, de subdesarrollo y desarrollo, y tampoco existe el concepto de riqueza y pobreza determinadas por la acumulación y carencia de bienes materiales. Continua Massiris afirmando que, para estas comunidades, el desarrollo se entiende en términos de "buen vivir" o "vivir bien"; estos conceptos rompen todos los paradigmas capitalistas de la forma actual de vivir y de consumir sin control, pues cambian su forma de actuar y de pensar para 
defender una concepción basada en estados de plenitud simultáneos y colectivos, referidos al goce de las libertades, al ejercicio de los derechos, al cumplimiento de los deberes y a la satisfacción de las necesidades, en paz y armonía con la naturaleza y entre todos.

No puede haber desarrollo, es decir, no se puede vivir bien si otros viven mal, como tampoco se puede vivir bien si la satisfacción de las necesidades implica la destrucción de la naturaleza. Se trata del acceso $\mathrm{y}$ del disfrute de los bienes materiales y de la realización efectiva, subjetiva, intelectual y espiritual, en armonía con la naturaleza y en comunidad con los seres humanos; muy distinto de un vivir mejor occidental que es separado de los demás e inclusive a expensas de los demás y separado de la naturaleza (República de Bolivia, 2006, citado por Massiris A., 2012). Por tanto, la localización de nuevas áreas de enterramiento de residuos sólidos no corresponde a un vivir bien, pues afecta los recursos naturales y a la calidad de vida del área de influencia de este tipo de instalaciones no deseables.

Finalmente, es significativo resaltar lo que sostiene Massiris (2012) cuando plantea que la territorialidad y sostenibilidad del desarrollo inherente al Desarrollo Territorial Sostenible se debe entender desde una perspectiva socioterritorial, en cuanto aprovechamiento ecológicamente sostenible de los recursos naturales y de mantenimiento de las condiciones ambientales propicias para una vida sana en el largo plazo, y en cuanto la superación de la pobreza, la exclusión social y la fragmentación territorial. Es decir, se trata de un desarrollo con sentido humano, ecológico y territorial. Así, pues, la opción de la disposición final de residuos vía relleno sanitario debe ser reevaluada y repensada por parte del Estado para que los ciudadanos, no solo del Distrito Capital, sino del país, puedan vivir bien con justicia ambiental y justicia social como corresponde.

\section{Referencias}

Calderón, L. M. (2010). Formulación de una estrategia de gestión para los residuos sólidos domésticos en Colombia, basada en el modelo normativo, sistémico e institucional aplicado en Suecia. (Trabajo de grado, Universidad de La Salle). Recuperado de: http://repository. lasalle.edu.co/bitstream/handle/10185/14421/T41.08\%20C127f.pdf?sequence=1 
Claro, E. (2001). El rol de la compensación en la localización de rellenos sanitarios en Santiago. Ambiente y desarrollo, XVII(2),27-34.

Congreso de Colombia. (18 de julio de 2007). Ley 388. Por la cual se modifica la Ley 9 de 1989, y la Ley 2 de 1991 y se dictan otras disposiciones. [Ley 388 de 2007].

Contraloría de Bogotá, D.C. (2012, diciembre). Dictamen de auditoría gubernamental con enfoque integral modalidad especial, para el contrato 344 de 2010 en cuanto a la capacidad del Relleno Sanitario Doña Juana -RSDJ- y la planta de tratamiento de lixiviados PTL-. Recuperado de: http://www.contraloriabogota.gov.co/intranet/contenido/informes/ AuditoriaGubernamental/H\%C3\%A1bitat\%20y\%20Ambiente/PAD_2012/CicloIII/ Especial/UAESP.pdf

DNP - Departamento Nacional de Planeación. Documento. (2008) Conpes 3530. Lineamientos y estrategias para fortalecer el servicio público de aseo en el marco de la gestión integral de residuos sólidos. [Conpes 3530, 2008]. Recuperado de: https://www.minambiente.gov.co/ images/normativa/conpes/2008/conpes_3530_2008.pdf

Gómez, M., \& Gustavo-Delgado Mahecha, O. (1998). Espacio, territorio y región: conceptos básicos para un proyecto nacional. Recuperado de: http://acoge2000.homestead.com/files/ Montanez_y_Delgado._1998.pdf

Grajales-Quintero, A., Serrano-Moya, E. D., \& Hahn Von-H, C. M. (2013). Los métodos y procesos multicriterio para la evaluación. Luna Azul, (36), 285-306.

Hernández-Gómez, A., Rojas-Robles, R., \& Sánchez-Calderón, F. V. (2013). Cambios en el uso del suelo asociados a la expansión urbana y la planeación en el corregimiento de Pasquilla, zona rural de Bogotá (Colombia). Cuadernos de Geografía-Revista Colombiana de Geografia, 22(2), 257-271.

Massiris-Cabeza, A. (2012). Gestión territorial y desarrollo: hacia una política de desarrollo territorial sostenible en América Latina. Tunja: Universidad Pedagógica y Tecnológica de Colombia.

MAVDT - Ministerio de Ambiente, Vivienda y Desarrollo Territorial. (2005, marzo 23). Decreto 838. por el cual se modifica el Decreto 1713 de 2002 sobre disposición final de residuos sólidos y se dictan otras disposiciones [Decreto 838 de 2005]. DO: 45862.

Molina, D. C. M. (2008). El papel de la geografía en los conflictos ambientales urbanos. En G. A. Rodríguez, B. Londoño Toro \& G. J. Herrera Carrascal (Eds.), Ciudades ambientalmente sostenibles (pp. 281-303). Bogotá: Editorial Universidad del Rosario.

Noguera, K., \& Olivero, J. (2010). Los rellenos sanitarios en Latinoamérica: caso colombiano. Revista de la Academia Colombiana de Ciencias Exactas, Físicas y Naturales, 34(132), 347-356. 
Sendra, J. B., Muñoz, M. Á. D., Durán, A. E. R., \& García, M. J. S. (2000). La componente geográfica en la percepción pública de las actividades no deseadas: las instalaciones para el tratamiento de residuos en el área metropolitana de Madrid. Lecturas geográficas, 1015.

Santos, M. (1997). Temica, Espap, Tempo. Globalizao emeio temico-cientifico infmmbonal. Siio Paulo: Editora Hucitec.

Recepción: 6 de febrero de 2015

Evaluación: 15 de marzo de 2015

Aceptación: 26 de abril de 2015 